\title{
Search for the QCD critical point at SPS energies
}

\section{Katarzyna Grebieszkow* for the NA49 tand the NA61/SHINE Collaborations}

Warsaw University of Technology

E-mail: kperleif.pw.edu.pl

Lattice QCD calculations locate the QCD critical point at energies accessible at the CERN Super Proton Synchrotron (SPS). We present average transverse momentum and multiplicity fluctuations, as well as baryon and anti-baryon transverse mass spectra which are expected to be sensitive to effects of the critical point. The future CP search strategy of the NA61/SHINE experiment at the SPS is also discussed.

European Physical Society Europhysics Conference on High Energy Physics, EPS-HEP 2009,

July 16 - 222009

Krakow, Poland

\footnotetext{
* Speaker.

${ }^{\dagger}$ See http://na49info.web.cern.ch/na49info/na49/Collaboration/authors.html

${ }^{\ddagger}$ See https://na61.web.cern.ch/na61/xc/index.html?O=authors
} 


\section{Introduction and motivation}

Theoretical calculations suggest that the critical point (CP) of strongly interacting matter may be accessible in the SPS energy range [1]. We have studied event-by-event average $p_{T}$ and multiplicity fluctuations, as well as transverse mass spectra of baryons and anti-baryons which are suggested observables sensitive to effects of the $\mathrm{CP}$ in ultra-relativistic heavy ion collisions.

The effects are expected to be maximal when freeze-out happens near the critical point. The position of chemical freeze-out point in the $\left(T-\mu_{B}\right)$ diagram can be varied by changing the energy and the size of the colliding system (Fig. 11). Therefore we have analyzed in NA49 [2] the energy dependence of the proposed $\mathrm{CP}$ sensitive observables for central $\mathrm{Pb}+\mathrm{Pb}$ collisions (beam

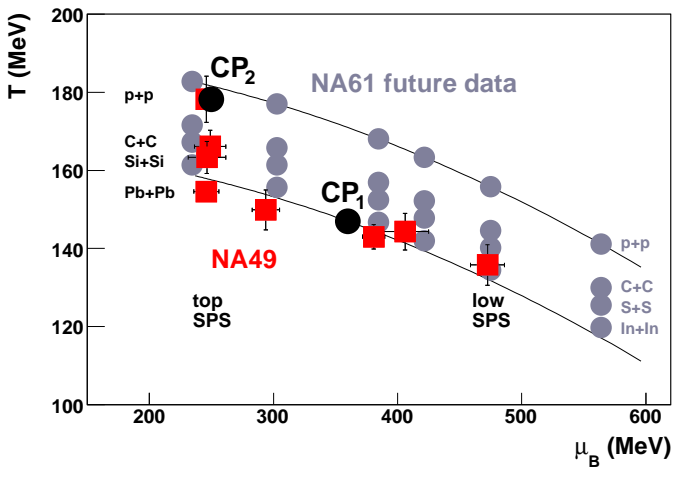

Figure 1: Chemical freeze-out points in NA49 (red) and those expected in NA61 (violet). $C P_{1}$ and $C P_{2}$ were considered in NA49 as possible locations of the critical point: $\mu_{B}\left(C P_{1}\right)$ from lattice QCD calculations [1] and $C P_{2}$ assuming that the chemical freeze-out point of $\mathrm{p}+\mathrm{p}$ data at $158 \mathrm{~A}$ $\mathrm{GeV}$ may be located on the phase transition line. energies $20 A-158 A \mathrm{GeV}$ ), and their system size dependence ( $\mathrm{p}+\mathrm{p}, \mathrm{C}+\mathrm{C}, \mathrm{Si}+\mathrm{Si}$, and $\mathrm{Pb}+\mathrm{Pb}$ ) at the highest SPS energy.

\section{Event-by-event average $p_{T}$ and multiplicity fluctuations}

Enlarged event-by-event fluctuations of multiplicity $\mathrm{N}$ and mean $p_{T}$ were suggested as a signature of the critical point [3]. The NA49 experiment used the $\Phi_{p_{T}}$ correlation measure [4, 5] and the scaled variance of the multiplicity distribution $\omega$ [6, 而] to study average $p_{T}$ and $N$ fluctuations, respectively. For $\omega$, we selected very central collisions only (1\% most central) due to its strong dependence on fluctuations of the number of participants $N_{\text {part }}$.

The energy $\left(\mu_{B}\right)$ dependence of $\Phi_{p_{T}}$ and $\omega$ together with predictions for $C P_{1}$ were presented at this conference (see also [8]). The NA49 data show no significant peak in the energy dependence of $\Phi_{p_{T}}$ and $\omega$ at SPS energies thus providing no indications of the critical point at $C P_{1}$ (see Fig. 1).

Figures 2 and 3 present the system size $\left(T_{c h e m}{ }^{1}\right)$ dependence of $\Phi_{p_{T}}$ and $\omega$. The lines correspond to predictions for $C P_{2}$ (see Fig. 1) with estimated magnitude of the effects ${ }^{2}$ for $\Phi_{p_{T}}$ and $\omega$ at $C P_{2}$ taken from Ref.[3, 9] assuming correlation lengths $\xi$ decreasing monotonically with decreasing system size: a) $\xi(\mathrm{Pb}+\mathrm{Pb})=6 \mathrm{fm}$ and $\xi(\mathrm{p}+\mathrm{p})=2 \mathrm{fm}$ (dashed lines) or b) $\xi(\mathrm{Pb}+\mathrm{Pb})=3$ $\mathrm{fm}$ and $\xi(\mathrm{p}+\mathrm{p})=1 \mathrm{fm}$ (solid lines). The width of the enhancement due to $\mathrm{CP}$ in the $\left(T, \mu_{B}\right)$ plane is based on Ref. [12] and taken as $\sigma(T) \approx 10 \mathrm{MeV}$. A maximum of $\Phi_{p_{T}}$ and $\omega$ is observed for $\mathrm{C}+\mathrm{C}$ and $\mathrm{Si}+\mathrm{Si}$ interactions at the top SPS energy. It is two times higher for all charged than for negatively charged particles, as expected for the effect of the CP [3]. Results presented in Figs. 2 and 3 suggest that the NA49 data are consistent with $\mathrm{CP}_{2}$ predictions.

\footnotetext{
${ }^{1} T_{\text {chem }}$ values were taken from fits of the hadron gas model [1] to particle yields.

${ }^{2}$ Predicted magnitudes include corrections by NA49 due to the limited rapidity range (forward-rapidity: $y_{\pi}^{*}>1.1$ ) and azimuthal angle acceptance of the detector (for details see corresponding NA49 papers).
} 


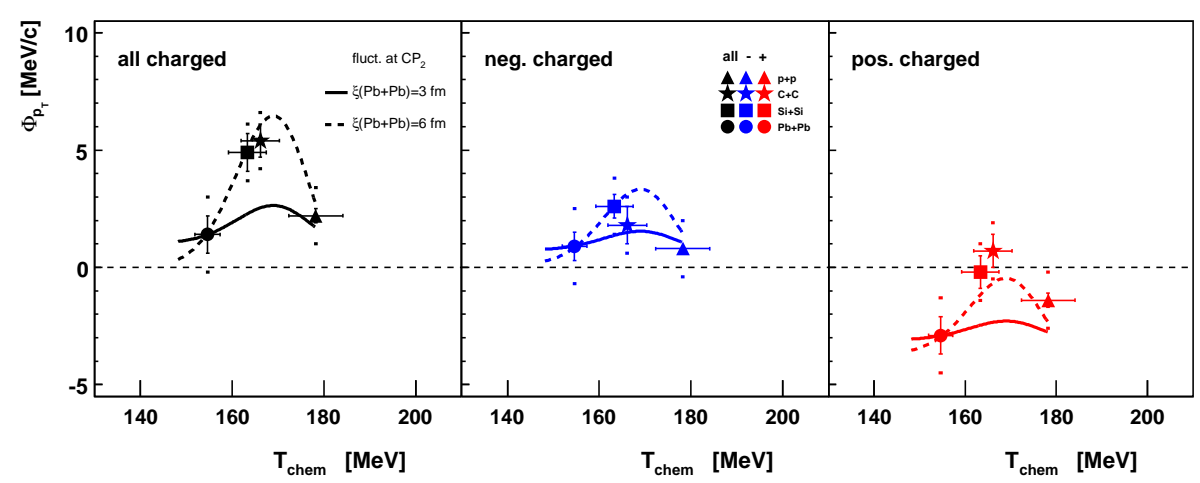

Figure 2: System size dependence of $\Phi_{p_{T}}$ at $158 \mathrm{~A} \mathrm{GeV}$ (forward rapidity, NA49 azimuthal angle acceptance) showing results from $\mathrm{p}+\mathrm{p}$, semi-central $\mathrm{C}+\mathrm{C}(15.3 \%)$ and $\mathrm{Si}+\mathrm{Si}(12.2 \%)$, and $5 \%$ most central $\mathrm{Pb}+\mathrm{Pb}$ collisions [卂]. Lines correspond to $C P_{2}$ predictions (see text) shifted to reproduce the $\Phi_{p_{T}}$ value for central $\mathrm{Pb}+\mathrm{Pb}$ collisions.

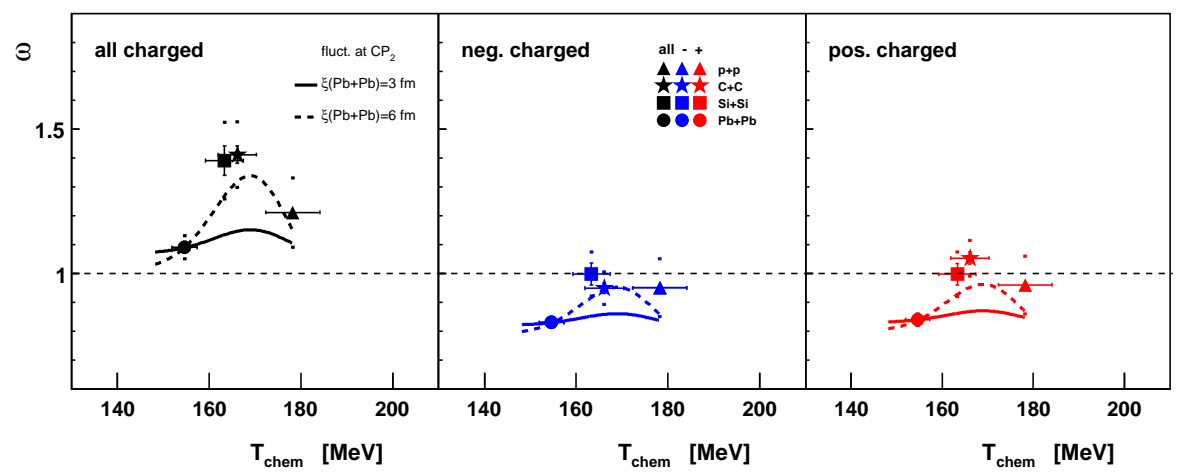

Figure 3: System size dependence of $\omega$ at $158 \mathrm{~A} \mathrm{GeV} \mathrm{(forward} \mathrm{rapidity,} \mathrm{NA49} \mathrm{azimuthal} \mathrm{angle} \mathrm{acceptance)}$ for the $1 \%$ most central $\mathrm{p}+\mathrm{p}\left[\right.$ [6], $\mathrm{C}+\mathrm{C}$ and $\mathrm{Si}+\mathrm{Si}[10]$, and $\mathrm{Pb}+\mathrm{Pb}$ collisions 何. Lines correspond to $\mathrm{CP}_{2}$ predictions (see text) shifted to reproduce the $\omega$ value for central $\mathrm{Pb}+\mathrm{Pb}$ collisions.

It is expected that fluctuations due to the $\mathrm{CP}$ originate mainly from low $p_{T}$ pions [3]. Therefore the NA49 analysis of $\Phi_{p_{T}}$ was performed also for two separate $p_{T}$ regions (Figs. 7 and 5). Indeed, the high $p_{T}$ region shows fluctuations consistent with zero (Fig. 4) and correlations are observed predominantly at low $p_{T}$ (Fig. 5). However, in low $p_{T}$ region, data do not show a maximum of $\Phi_{p_{T}}$, but a continuous rise towards $\mathrm{Pb}+\mathrm{Pb}$ collisions. The origin of this behavior is currently being analyzed (short range correlations are considered).

\section{Transverse mass spectra of baryons and anti-baryons}

It was suggested [13] that the critical point serves as an attractor of hydrodynamical trajectories in the $\left(T, \mu_{B}\right)$ phase diagram. This was conjectured to lead to a decrease of the anti-baryon to baryon $(\bar{B} / B)$ ratio with increasing transverse momentum. The $\bar{p} / p, \bar{\Lambda} / \Lambda$, and $\bar{\Xi}^{+} / \Xi^{-}$ratios versus reduced transverse mass $m_{T}-m_{0}$ were studied by the NA49 experiment [8] and presented at this conference. The slopes of all three $\bar{B} / B$ ratios show no significant energy dependence, thus implying that transverse mass spectra of $B$ and $\bar{B}$ do not provide evidence for the critical point in the SPS energy range. 


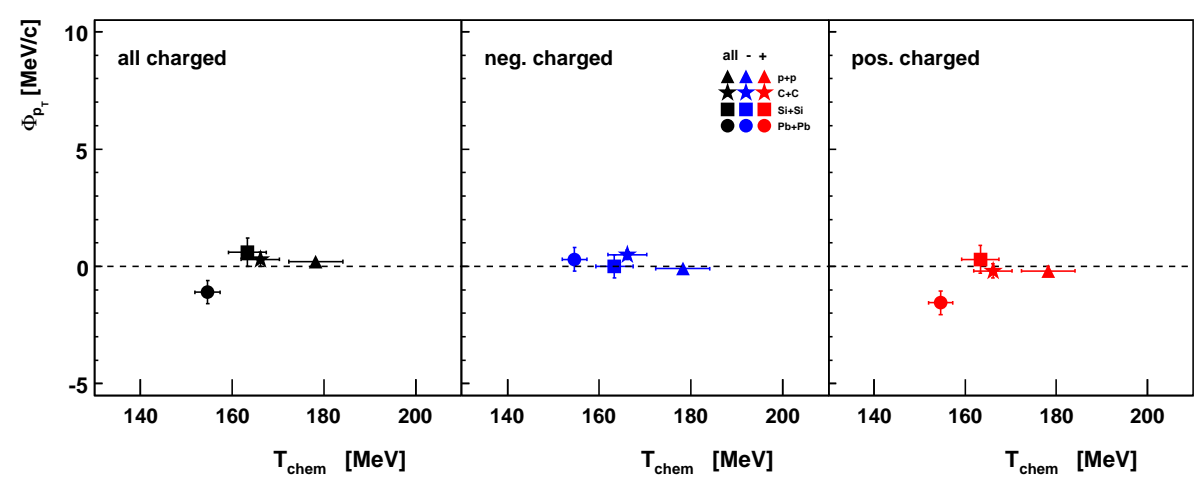

Figure 4: The same as Fig. 目, but for high- $p_{T}$ particles $\left(0.5<p_{T}<1.5 \mathrm{GeV} / \mathrm{c}\right)$.

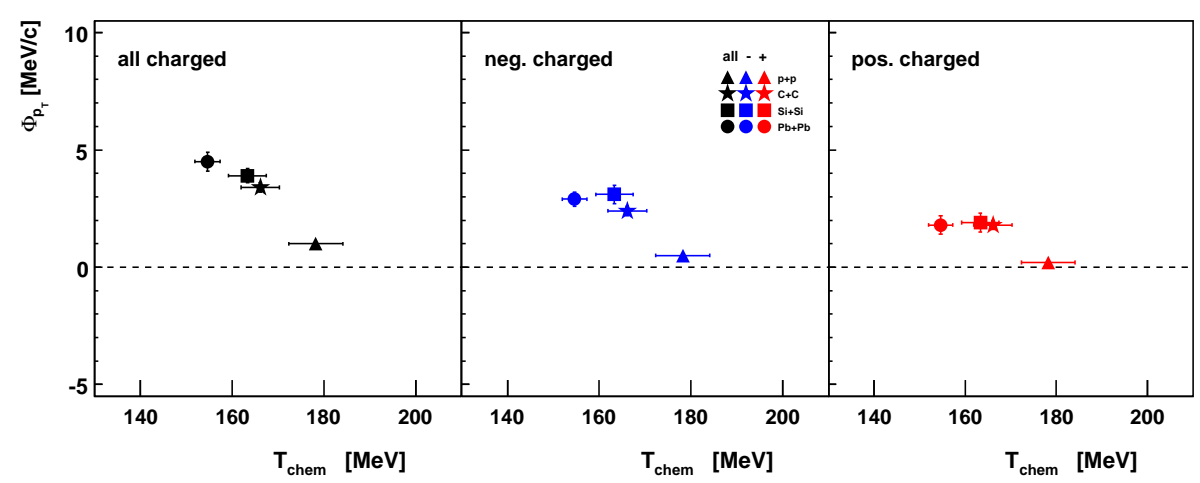

Figure 5: The same as Fig. 2, but for low- $p_{T}$ particles $\left(0.005<p_{T}<0.5 \mathrm{GeV} / \mathrm{c}\right)$.

\section{Summary of NA49 results and strategy of NA61/SHINE}

The energy dependence of average $p_{T}$ and multiplicity fluctuations, and ratios of the antibaryon/baryon transverse mass spectra in central $\mathrm{Pb}+\mathrm{Pb}$ collisions provide no indications of the critical point. The system size dependence at $158 \mathrm{~A} \mathrm{GeV}$ exhibits a maximum of mean $p_{T}$ and multiplicity fluctuations in the complete $p_{T}$ range (consistent with $C P_{2}$ predictions) and an increase (from $\mathrm{p}+\mathrm{p}$ up to $\mathrm{Pb}+\mathrm{Pb}$ ) of mean $p_{T}$ fluctuations in the low $p_{T}$ region. The low $p_{T}$ region will be carefully analyzed for the effects of short range correlations on $\Phi_{p_{T}}$ and $\omega$.

A detailed energy and system-size scan is necessary to establish the existence of the critical point. Therefore the CP search will be continued by the NA61/SHINE [14] experiment which is based on the upgraded NA49 detector. We plan to perform a two-dimensional scan with lighter ions $(\mathrm{p}+\mathrm{p}, \mathrm{C}+\mathrm{C}, \mathrm{S}+\mathrm{S}, \mathrm{In}+\mathrm{In})$ in a broad beam energy range $(10 \mathrm{~A}-158 \mathrm{~A} \mathrm{GeV})$. The hypothetical chemical freeze-out points in the NA61 experiment are presented in Fig. 1.Together with existing NA49 data the scan may help to locate the QCD critical point in the $\left(T, \mu_{B}\right)$ phase diagram.

\section{References}

[1] Z. Fodor and S. D. Katz, Critical point of $Q C D$ at finite $T$ and $\mu$, lattice results for physical quark masses, JHEP 0404, 050 (2004) [hep-lat/ 040200 6]. 
[2] S. Afanasiev et al. (NA49 Collab.), The NA49 large acceptance hadron detector, Nucl. Instrum. Meth. A430, 210 (1999).

[3] M. Stephanov, K. Rajagopal, and E. V. Shuryak, Event-by-event fluctuations in heavy ion collisions and the QCD critical point, Phys. Rev. D60, 114028 (1999) [hep-ph/9903292].

[4] T. Anticic et al. (NA49 Collab.), Transverse Momentum Fluctuations in Nuclear Collisions at 158 AGeV, Phys. Rev. C70, 034902 (2004) [hep-ex/0311009].

[5] T. Anticic et al. (NA49 Collab.), Energy dependence of transverse momentum fluctuations in $\mathrm{Pb}+\mathrm{Pb}$ collisions at the CERN Super Proton Synchrotron (SPS) at 20A to 158A GeV, Phys. Rev. C79, 044904 (2009) [arXiv:0810.5580].

[6] C. Alt et al. (NA49 Collab.), Centrality and system size dependence of multiplicity fluctuations in nuclear collisions at 158A GeV, Phys. Rev. C75, 064904 (2007) [nucl-ex/0612010].

[7] C. Alt et al. (NA49 Collab.), Energy Dependence of Multiplicity Fluctuations in Heavy Ion Collisions at the CERN SPS, Phys. Rev. C78, 034914 (2008) [arXiv: 0712.3216].

[8] K. Grebieszkow et al. (NA49 Collab.), Search for the critical point of strongly interacting matter in NA49, Nucl. Phys. A830, 547C (2009) [arXiv:0 907.4101$].$

[9] M. Stephanov, private communication.

[10] B. Lungwitz, PhD thesis (2008), https://edms.cern.ch/document/989055/1 (unpublished).

[11] F. Becattini, J. Manninen, M. Gazdzicki, Energy and system size dependence of chemical freeze-out in relativistic nuclear collisions, Phys. Rev C73, 044905 (2006) [hep-ph / 0511092].

[12] Y. Hatta and T. Ikeda, Universality, the QCD critical/tricritical point and the quark number susceptibility, Phys. Rev. D67, 014028 (2003) [hep-ph/0210284].

[13] Askawa, et al., Transverse Velocity Dependence of the Proton-Antiproton Ratio as a Signature of the QCD Critical Point, Phys. Rev. Lett 101, 122302 (2008) [arXiv: 0803.244 9];

Luo et al., Signature of QCD critical point: Anomalous transverse velocity dependence of antiproton-proton ratio, Phys. Lett. B673, 268 (2009) [arXiv: 0903.0024 ].

[14] https://na61.web.cern.ch/na61/xc/index.html 